\title{
Prácticas agroecológicas, micorrización y productividad del intercultivo maíz - soya (Zea mays L. - Glycine max L.)
}

\author{
Agroecological practices, michorrization and productivity of intercropping \\ corn - soybean (Zea mays L. - Glycine max L.)
}

Oscar Eduardo Sanclemente Reyes ${ }^{1 *}$, Marina Sánchez de Prager $^{2}$, Martín Prager Mosquera $^{2}$

\section{RESUMEN}

Se evaluó la influencia de prácticas agroecológicas sobre micorrización y rendimiento del intercultivo maíz-soya, en un Typic Haplustert del Centro Experimental de la Universidad Nacional de Colombia (Candelaria - Colombia). En 2011-II, se sembró Mucuna pruriens - maíz cuyos residuos vegetales se usaron como abono verde (AV) o acolchado orgánico (AO). En 2012-I, se sembró en rotación maíz- soya en un diseño experimental en parcelas divididas (AV, AO) y barbecho natural (BA), con tres réplicas. Se usó compost, fertilizante NPK (15-15-15) y un control sin fertilización, en subparcelas. En floración, se evaluó la longitud micelial de hongos micorrícicos arbusculares (HMA) en la raíz, y su aporte de $\mathrm{P}$ a la planta. Finalmente, se estimó el rendimiento de grano seco. Se observó que el AO incrementó significativamente ( $<<0,05)$ la actividad de HMA $(29,7 \%)$ y el rendimiento en soya, por liberación gradual de nutrientes. El uso de NPK en subparcelas redujo la actividad de HMA comparado con el manejo sin fertilización adicional, afectando la disponibilidad de $\mathrm{P}$ en el intercultivo por esta vía simbiótica. El análisis de componentes indicó que el uso de $\mathrm{AO}$, con o sin compost, benefició tanto la actividad micorrícica como la productividad del intercultivo maízsoya. Estos resultados muestran las bondades del uso de prácticas agroecológicas sobre la sostenibilidad del recurso suelo y la productividad de cultivos tradicionales.

Palabras clave: Simbiosis rizosférica, abonos verdes, acolchados orgánicos, ciclaje de nutrientes, Mucuna pruriens

\begin{abstract}
The influence of agroecological practices, michorrization and yield of intercropping corn-soybean in a Typic Haplustert of the Experimental Center of the National University of Colombia (Candelaria-Colombia) was evaluated. In 2011-II, Mucuna prurienscorn was planted and its plant residues were used as green manure (GM) or mulch (OM). In 2012-I, corn-soybean was planted in rotation on experimental design in split plots (GM, OM) and natural fallow (FA), with three replicates. Composting, fertilizer NPK (15-15-15) and a control without fertilization, was used in subplots. At flowering, the mycelial length of arbuscular mycorrhizal fungi AMF and its $P$ contribution to plant was assessed. Finally, dry grain yield was estimated. It was noted that the ML increased significantly $(p<0,05)$ activity of AMF (29,7\%), and the soybean yield by gradual release of nutrients. The use of NPK in subplots, reduced AMF activity compared with no additional fertilization management, affecting the availability of $P$ in the intercropping by this symbiotic way. The component analysis indicated that OM used with or without composting, benefited both mycorrhizal activity and productivity of corn-soybean intercropping. These results show the benefits of the use of agroecological practices on sustainability of soil resources and productivity of traditional crops.
\end{abstract}

Keywords: Symbiosis rhizosphere, green manures, organic mulches, nutrient cycling, Mucuna pruriens

\section{Introducción}

Los cultivos de maíz y soya han sido históricamente importantes para la alimentación mundial, debido a su aporte energético y proteico. En la actualidad, cerca de 3 billones de personas derivan su nutrición directamente de estos dos cultivos, elevando ostensiblemente la producción industrial de estos monocultivos y generando efectos socio-económicos y ambientales de importancia (Rosset y Ávila, 2008; Altieri y Nicholls, 2012). La mayoría de estos resultados

\footnotetext{
Programa de Ingeniería Ambiental en la Universidad Nacional Abierta y a Distancia- Colombia.

2 Departamento de Ciencias Biológicas en la Universidad Nacional de Colombia, Sede. Palmira, Colombia.

* Autor por correspondencia: oscar.sanclemente@unad.edu.co
}

Fecha de Recepción: 14 febrero, 2017.

Fecha de Aceptación: 8 mayo, 2018.

DOI: http://dx.doi.org/10.4067/S0718-34292018005000301. Publicado en línea: 25-julio-2018. 
provienen de la derivación de estos productos hacia la industria, el uso de mecanización y la aplicación excesiva de fertilizantes de síntesis, pesticidas, fungicidas y herbicidas derivados del petróleo, que generan efectos nocivos sobre el suelo y los recursos hídricos (Altieri et al., 2012; Sanclemente, 2015).

La Agroecología, como ciencia, estudia los agroecosistemas desde el punto de vista de las interacciones ecológicas y culturales (León, 2009), con una mirada a largo plazo, considerando las alternativas generadas en el largo proceso evolutivo que permitió el establecimiento de la vida en todas sus manifestaciones y su permanencia sobre la tierra. Desde la perspectiva de los ecosistemas y agroecosistemas, las micorrizas arbusculares (MA) se definen como una íntima relación entre las raíces de la mayoría de las plantas y algunos hongos del suelo del género Glomeromycota sp. (Sieverding, 1991; Sánchez de Prager et al., 2007; Siqueira, 2010; Oehl et al., 2011; Prager et al., 2012). A través de esta simbiosis, se proveen servicios ecológicos a las plantas, tales como el incremento en la absorción de algunos nutrientes de difícil movilidad en el suelo ( $\mathrm{P}, \mathrm{Fe}$, entre otros), debido a la acción del micelio externo del hongo micorrícico arbuscular HMA ligado a los sistemas radicales de las plantas, mayor absorción de agua, de $\mathrm{N}, \mathrm{K}$ y algunos micronutrientes. Al mismo tiempo, mejora la sanidad vegetal. En soya, se ha encontrado estrecha relación entre la absorción de $\mathrm{P}$ en plantas micorrizadas y el incremento en la fijación de $\mathrm{N}_{2}$ por asociación con rizobios del suelo (Meghvansi et al., 2008; Juge et al., 2012).

Algunos estudios demuestran que el uso de fertilizantes de síntesis industrial reduce ostensiblemente los beneficios de la simbiosis por excesiva disponibilidad de nutrientes, afectando así la economía del cultivo y de los agricultores (Ipsilantis et al., 2012). Si bien existen tecnologías agroecológicas para el manejo de la fertilidad del suelo, tales como uso de compost, abonos verdes (AV) y acolchados orgánicos (AO), es necesario determinar la influencia que estas ejercen sobre el desarrollo de la MA en sistemas de policultivo gramínea-leguminosa, como es el caso maíz-soya. Estas investigaciones proveerán resultados que confirmarán los beneficios del uso de recursos orgánicos como estrategia para aportar nutrientes, mantener o mejorar la materia orgánica del suelo MOS y favorecer la economía del cultivo, al permitir la expresión de recursos microbiológicos que coevolucionaron con las plantas. El objetivo de este trabajo fue evaluar la influencia de estas tecnologías de manejo agroecológico del suelo, sobre la micorrización y el rendimiento de grano seco del intercultivo maíz-soya.

\section{Materiales y métodos}

El ensayo se desarrolló durante los meses de agosto de 2011 y julio de 2012, en el Centro Experimental de la Universidad Nacional de Colombia, sede Palmira, ubicado en el municipio de Candelaria - Valle del Cauca ( 990 msnm, $3^{\circ} 24^{\prime}$ latitud $\mathrm{N}$ y $76^{\circ} 26^{\prime}$ longitud O). El suelo es un Typic Haplustert francoso fino, que estuvo en descanso agrícola durante ocho años. Las características iniciales del horizonte arable del suelo (de 0-20 cm) fueron: $\mathrm{pH}(6,8), \operatorname{MOS}\left(26,6 \mathrm{~g} \cdot \mathrm{kg}^{-1}\right), \mathrm{N}$-total $(1,1$ $\left.\mathrm{g} \cdot \mathrm{kg}^{-1}\right)$, P-total $\left(57,4 \mathrm{~g} \cdot \mathrm{kg}^{-1}\right), \mathrm{CIC}\left(21,1 \mathrm{cmol} \cdot \mathrm{kg}^{-1}\right)$, $\mathrm{K}\left(0,44 \mathrm{cmol} \cdot \mathrm{kg}^{-1}\right), \mathrm{Ca}\left(10,9 \mathrm{cmol} \cdot \mathrm{kg}^{-1}\right), \mathrm{Mg}(5,3$ $\left.\mathrm{cmol} \cdot \mathrm{kg}^{-1}\right), \mathrm{Na}\left(0,10 \mathrm{cmol} \cdot \mathrm{kg}^{-1}\right)$. Durante el mes de agosto de 2011 se estableció el intercultivo maíz (Zea mays L.) - frijol terciopelo (Mucuna pruriens L.), reconocido por el sinergismo que expresa con maíz y la alta producción de biomasa fresca. Una vez cosechado el maíz choclo, 90 días después de la siembra, los residuos vegetales del intercultivo se adicionaron en la superficie (acolchado orgánico - AO), o fueron incorporados a una profundidad de $0-10 \mathrm{~cm}$ (abono verde- AV) mediante uso de motocultor. Paralelo a las prácticas de AO y $\mathrm{AV}$, como tratamiento control se adicionaron en superficie los residuos vegetales de Rottboelia cochinchinensis (Lour.) por ser el barbecho (BA) predominante en la zona.

Veinte (20) días después de la adición de los residuos vegetales se sembró el intercultivo maíz - soya en parcelas de $20 \mathrm{~m}^{2}$ a razón de 60 y 250 mil plantas $\cdot$ ha $^{-1}$, respectivamente. Adicionalmente, se comparó la fertilización de tipo orgánico con la fertilización basada en insumos de síntesis industrial. Se utilizó un diseño experimental de bloques completos al azar (BCAA) con arreglo en parcelas divididas y tres repeticiones, usando el manejo de los residuos vegetales de cosecha como tratamiento en las parcelas principales y la fertilización complementaria como tratamiento en las subparcelas (Tabla 1).

En estado fenológico de floración de los intercultivos, se evaluó la influencia de los 
Tabla 1. Descripción de los tratamientos probados en el ensayo.

\begin{tabular}{ccccc}
\hline \multirow{2}{*}{ Tratamiento } & \multicolumn{2}{c}{ Parcela Principal* } & \multicolumn{2}{c}{ Subparcela** } \\
\cline { 2 - 5 } & Tipo & Dosis $\left(\mathbf{t} \cdot \mathbf{h a}^{-\mathbf{1}}\right)$ & Tipo & Dosis (t·ha-1) \\
\hline T1 & Abono verde & 4,17 & Compost & 3,40 \\
T2 & Abono verde & 4,17 & NPK (15-15-15) & 0,33 \\
T3 & Abono verde & 4,17 & Sin fertilizar & - \\
T4 & Acolchado & 4,17 & Compost & 3,40 \\
T5 & Acolchado & 4,17 & NPK (15-15-15) & 0,33 \\
T6 & Acolchado & 4,17 & Sin fertilizar & - \\
T7 & Barbecho & 2,24 & Compost & 3,40 \\
T8 & Barbecho & 2,24 & NPK (15-15-15) & 0,33 \\
T9 & Barbecho & 2,24 & Sin fertilizar & - \\
\hline
\end{tabular}

*Las dosis en las parcelas principales se expresan en toneladas de materia seca por hectárea. **Las dosis en subparcelas se expresan con los contenidos de humedad comercial de los productos y se escogieron con base en el aporte de $50 \mathrm{kgN} \cdot \mathrm{ha}^{-1}$ como requerimiento del intercultivo. El compost utilizado proviene de gallinaza, producido por la empresa Abonissa KIKES S.A.

tratamientos sobre la simbiosis rizosférica MA en el intercultivo a partir de las variables: longitud del micelio externo (LME), porcentaje de micelio vital (\%MV) y porcentaje de micelio externo activo (\%MEA). Para ello, se tomaron muestras en campo del tejido de raíces jóvenes y suelo rizosférico de $0-20 \mathrm{~cm}$, usando palín. En laboratorio, se estimó la LME mediante metodología descrita por Sánchez de Prager et al., (2010) y evaluando por método Tennant (1975) de intersección en cuadrantes a 200 aumentos en microscopio óptico (AmScope, T120B-3M), la cantidad de micelio en muestras de suelo retenido en filtros de nitrocelulosa. El \%MV y el \%MEA se estimó mediante tinción de succinato deshidrogenasa, en el tejido cortical y el micelio externo, respectivamente; como lo describen Sánchez de Prager et al. (2010). Cinco meses después de la siembra, se cosecharon los granos secos de maíz y soya, expresando el rendimiento a $14 \%$ de humedad gravimétrica. Los resultados se sometieron a análisis de varianza, comparación de medias con la prueba de Tukey $(\mathrm{p}<0,05)$ y técnica multivariada de componentes principales usando el paquete estadístico SAS versión 9.2 (2008).

\section{Resultados y discusión}

Las variables LME, \%MV y \%MEA de HMA se analizaron comparando el efecto en parcelas principales y subparcelas. Se observó mayor actividad de los HMA en maíz. Esto se puede deber a la mayor capacidad fotosintética del maíz por ser una planta de metabolismos $\mathrm{C} 4$, que le permite invertir mayor cantidad de fotoasimilados en la simbiosis, a lo cual se suma la arquitectura de su sistema radical (Urcelay y Battistella, 2007, Sánchez de Prager et al., 2007). Del análisis de valores alcanzados por las variables evaluadas se desprende que hubo alta variabilidad espacial, lo que impidió detectar diferencias significativas entre los tratamientos (Tabla 2).

La LME, el \%MV y el \%MEA fueron estadísticamente similares $(\mathrm{p}<0,05)$ para los tratamientos en maíz y en soya, con excepción del \%MEA de HMA en la parcela principal con AO en soya, la que obtuvo un $81,9 \%$, sin variar significativamente de $\mathrm{AV}(59,7 \%)$, pero sícon respecto al BA $(52,2 \%)$. Esto indica mayor transferencia de P a la planta por el micelio externo de los HMA en las parcelas a las cuales se adicionó material orgánico procedente del intercultivo maíz - frijol terciopelo independiente de su forma de aplicación, factor que incide sobre el metabolismo de la planta y el proceso de fijación de $\mathrm{N}_{2}$ que es altamente dependiente del suministro de $\mathrm{P}$ (Ojiem et al., 2007).

En la práctica de $\mathrm{AO}$, el aporte de nutrientes por adición de residuos vegetales en superficie es más lento que en el AV. Esto se debe a que los residuos vegetales tienen menor superficie de 
Tabla 2. Incidencia del manejo de los residuos vegetales en parcelas principales y subparcelas, sobre las variables LME, \%MV y \%MEA de HMA, en el intercultivo maíz-soya*.

\begin{tabular}{|c|c|c|c|c|c|c|}
\hline & $\begin{array}{c}\text { LME } \\
\left(\mathrm{m} \cdot \text { g suelo-1 }^{-1}\right)\end{array}$ & $\begin{array}{c}\text { MV } \\
\%\end{array}$ & $\begin{array}{c}\text { MEA } \\
\%\end{array}$ & $\begin{array}{c}\text { LME } \\
\left(\mathbf{m} \cdot \text { g suelo-1) }^{-1}\right.\end{array}$ & $\begin{array}{c}\text { MV } \\
\%\end{array}$ & $\begin{array}{c}\text { MEA } \\
\%\end{array}$ \\
\hline & \multicolumn{3}{|c|}{ 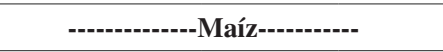 } & \multicolumn{3}{|c|}{-------------Soya------------- } \\
\hline \multicolumn{7}{|c|}{ Manejo de Residuos Vegetales: } \\
\hline (AO) Acolchado orgánico & $8,5 \mathrm{a}$ & $50,8 \mathrm{a}$ & 89,5 a & $3,3 \mathrm{a}$ & $42,8 \mathrm{a}$ & $81,9 \mathrm{a}$ \\
\hline (AV) Abono verde & $7,2 \mathrm{a}$ & $46,8 \mathrm{a}$ & $76,1 \mathrm{a}$ & $2,1 \mathrm{a}$ & $43,1 \mathrm{a}$ & $59,7 \mathrm{ab}$ \\
\hline (BA) Barbecho & $6,0 \mathrm{a}$ & $49,0 \mathrm{a}$ & $69,0 \mathrm{a}$ & $2,8 \mathrm{a}$ & $49,0 \mathrm{a}$ & $52,2 \mathrm{~b}$ \\
\hline \multicolumn{7}{|c|}{ Fertilización complementaria: } \\
\hline Compost & $6,9 \mathrm{a}$ & $47,5 \mathrm{a}$ & $74,4 \mathrm{a}$ & $2,5 \mathrm{a}$ & $45,8 \mathrm{a}$ & $66,1 \mathrm{a}$ \\
\hline NPK (15- 15- 15) & $7,2 \mathrm{a}$ & $50,3 \mathrm{a}$ & $77,6 \mathrm{a}$ & $2,8 \mathrm{a}$ & $46,8 \mathrm{a}$ & $67,2 \mathrm{a}$ \\
\hline Sin fertilizante & $7,7 \mathrm{a}$ & $48,4 \mathrm{a}$ & $82,7 \mathrm{a}$ & $2,9 \mathrm{a}$ & $42,2 \mathrm{a}$ & $60,6 \mathrm{a}$ \\
\hline
\end{tabular}

contacto con el suelo, limitando la mineralización de nutrientes. Adicionalmente, en el AO se busca sumar su alta capacidad de retención de agua y nutrientes por imbibición, factor que regula el uso de nutrientes solubles vía fertilización complementaria, según lo descrito por Gómez Zambrano (2011). Mediante la práctica de AO se puede potenciar la MA como mecanismo biológico para proveer agua y nutrientes superando la zona de agotamiento del suelo, a través de las redes y puentes miceliales de los HMA (Gosling et al., 2006; Deguchi et al., 2007). En esta investigación, con respecto a los otros tratamientos, el AO en maíz marcó una diferencia en longitud de micelio externo de HMA, de 1,3 y 2,5 m.g suelo-1, respectivamente $\mathrm{y}$, en soya esta diferencia fue de 0,5 y $1,2 \mathrm{~m} \cdot \mathrm{g} \mathrm{suelo}{ }^{-1}$, respectivamente. Esta ventaja del AO significa mayor volumen de suelo explorado con la MA, favoreciendo la nutrición y economía del intercultivo comercial (Sánchez de Prager et al., 2007).

En la fertilización complementaria con compost y fertilizante de síntesis, no se observaron diferencias estadísticas significativas $(\mathrm{p}<0,05)$ sobre las variables de HMA. Sin embargo, se encontró que la fertilización complementaria redujo la LME tanto en maíz como en soya. Esto puede deberse a la mayor disponibilidad de nutrientes solubles para absorción directa por las raíces, lo cual inhibe el crecimiento del micelio de HMA en el suelo, de acuerdo con lo planteado por distintos investigadores (Sánchez de Prager et al., 2009; Ipsilantis et al., 2012).

La influencia de la actividad de la MA y la absorción radical de agua y nutrientes finalmente se expresa en el rendimiento de grano del intercultivo (Figura 1). En maíz, a pesar de que el rendimiento en el ensayo estuvo entre 4.124 y $5.702 \mathrm{~kg} \cdot \mathrm{ha}^{-1}$, cercanos a la media local de 7.200 $\mathrm{kg} \cdot \mathrm{ha}^{-1}$ (FENALCE, 2013), no se observaron diferencias estadísticas significativas $(p<0,05)$ entre los tratamientos, debido a la alta variabilidad de los datos entre bloques.

En soya, con el uso de AO se obtuvo el mayor valor de rendimiento, seguido del AV y de BA. Los rendimientos en $\mathrm{AO}$ y $\mathrm{AO}+$ compost fueron los más altos con 2.466 y $2.345 \mathrm{~kg} \cdot \mathrm{ha}^{-1}$, superiores al promedio de la región de $1.800 \mathrm{~kg} \cdot \mathrm{ha}^{-1}$ (FENALCE, 2013). El control (barbecho sin fertilizar) presentó el rendimiento más bajo $\left(1.140 \mathrm{~kg} \cdot \mathrm{ha}^{-1}\right)$, que pudo deberse al reducido aporte de nutrientes y a la baja actividad de los HMA que incidieron sobre la absorción de $\mathrm{P}$ para el proceso productivo y la fijación simbiótica de $\mathrm{N}_{2}$.

La tabla 3 muestra el análisis de componentes principales para las variables evaluadas en el ensayo. En maíz, los tres primeros componentes explican el 98,0\% de la variación e indican que la LME, seguida del \%MEA, el \% MV y finalmente el rendimiento de grano fueron las variables más influyentes sobre la variabilidad total. En soya, los tres primeros componentes explican el 95,4\% de la variación e indican que el rendimiento de grano seco, seguido del \%MEA, la LME y finalmente el $\% \mathrm{MV}$ fueron las variables más influyentes sobre la variabilidad total.

Estos resultados confirman la alta respuesta de la longitud LME y actividad del micelio externo de los HMA - \%MEA - asociados a maíz 


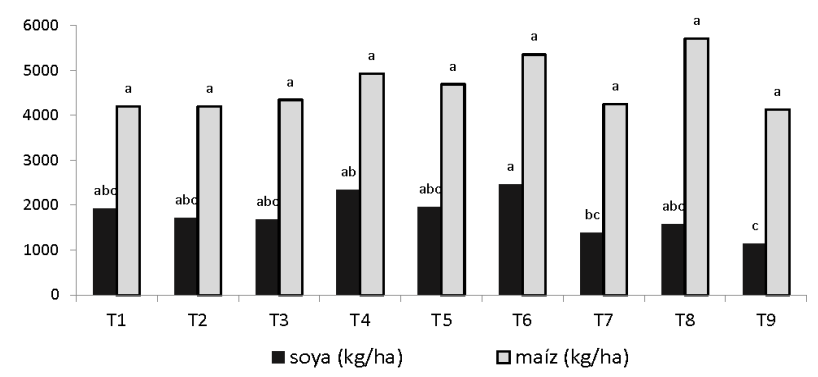

Figura 1. Rendimiento de grano seco de maíz y soya $\left(\mathrm{kg} \cdot \mathrm{ha}^{-1}\right)$ al finalizar el ensayo. El peso de grano se expresa con un contenido de humedad del 14\%. Las letras en las barras indican diferencias estadísticamente significativas entre los tratamientos en prueba de Tukey $(\mathrm{p}<0,05)$.

Tabla 3. Análisis de componentes principales para las variables evaluadas en maíz (M) y soya (S).

\begin{tabular}{lcccccc}
\hline \multicolumn{1}{c}{ Variables } & \multicolumn{2}{c}{ Componente 1 } & \multicolumn{2}{c}{ Componente 2 } & \multicolumn{2}{c}{ Componente 3 } \\
\hline Rendimiento & M & S & M & S & M & S \\
\%MV & $-0,350$ & $0,653^{*}$ & $0,584^{*}$ & 0,157 & $0,730^{*}$ & $-0,139$ \\
$\%$ MEA & $-0,069$ & $-0,315$ & $0,743^{*}$ & $0,671^{*}$ & $-0,640^{*}$ & $0,620^{*}$ \\
LME & $0,639^{*}$ & $0,621^{*}$ & 0,313 & $-0,160$ & 0,100 & $0,654^{*}$ \\
* Indica las variables de mayor peso en cada & $0,680^{*}$ & 0,295 & 0,082 & $0,706^{*}$ & 0,216 & $-0,408$ \\
\hline
\end{tabular}

para los distintos tratamientos. Sin embargo, esta tendencia no fue tan marcada para el caso de la actividad del \%MV de HMA que colonizó la raíz de la gramínea. De forma similar, no afectó el rendimiento de grano seco, lo que pudo deberse a la alta disponibilidad de $\mathrm{P}$ en el suelo que disminuyó la absorción del nutriente por intermedio del micelio de HMA y transporte hacia la planta por esta vía. En soya se registró mayor respuesta de los tratamientos sobre el rendimiento de grano seco, que tuvo alta relación con el $\%$ MEA, la LME y el \%MV, esta última variable de similar magnitud a la observada en maíz. Lo anterior indicaría que el aporte de nutrientes como el $\mathrm{P}$ a la leguminosa por acción de la MA estaría influyendo ostensiblemente en el proceso de fijación de $\mathrm{N}_{2}$.

La figura 2 ilustra la distribución de los tratamientos en los dos primeros componentes. En maíz, los tratamientos T2 (AV+NPK), T4 (AO+ compost), T5 (AO+ NPK) y T6 (AO) presentan tendencias similares al potenciar el desarrollo del micelio externo de HMA, su actividad sobre la absorción de P desde la solución del suelo y transferencia a la planta. La influencia de los tratamientos $\mathrm{T} 1$ (AV+ compost) y $\mathrm{T} 7$ (BA+ compost) sobre el desarrollo de la MA y la absorción de $\mathrm{P}$ no fue determinante, lo que explica su baja productividad en cosecha.

En soya, coincidente con maíz, en los tratamientos $\mathrm{T} 4(\mathrm{AO}+$ compost) y $\mathrm{T} 6(\mathrm{AO})$, la adición de los residuos vegetales en superficie incidió positivamente sobre el rendimiento de grano, la actividad del micelio externo de HMA y la absorción de P del suelo. En los tratamientos T1 (AV+ compost), $\mathrm{T} 2$ (AV+ NPK) y T3 (AV), la incorporación de los residuos vegetales al suelo influyó marginalmente sobre la actividad del micelio externo de HMA y su efecto en la absorción de P desde la solución. Lo anterior constituyó factor incidente sobre la baja productividad de granos por deficiencia en la absorción de nutrientes. Similar tendencia se observó en T7 (BA+ compost) y el control T9 (BA), los cuales obtuvieron los menores rendimientos del ensayo.

La práctica de $\mathrm{AV}$ pudo incrementar la mineralización de compuestos orgánicos presentes 


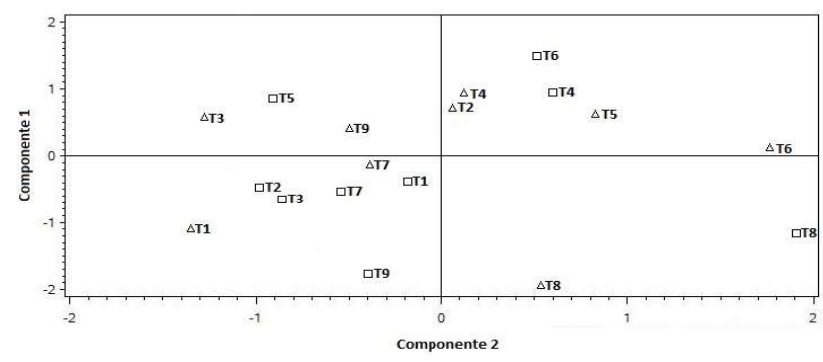

Figura 2. Distribución de los tratamientos en los dos primeros componentes principales. Maíz $(\Delta)$ y Soya $(\square)$.

en los residuos vegetales por mayor contacto con los microorganismos del suelo, ocasionando mayores pérdidas de nutrientes por volatilización, erosión y/o lixiviación comparadas con la práctica de $\mathrm{AO}$, como lo describen Sanclemente y Prager (2009). El bajo aporte de nutrientes en el tratamiento control afectó negativamente el rendimiento del intercultivo, al igual que la MA por su baja capacidad de inversión de fotoasimilados en la simbiosis. En T8 (BA+ NPK) se obtuvieron altos rendimientos de grano en maíz y bajos en soya. La gramínea mostró mayor capacidad de absorción de nutrientes solubles e incidencia negativa sobre la actividad simbiótica de HMA y en la leguminosa sobre la fijación de $\mathrm{N}_{2}$, proceso altamente sensible a la presencia de nitratos en solución (Salvagiotti et al., 2008; Lau et al., 2012).

Los resultados obtenidos señalan que el uso de prácticas de adición e incorporación de residuos orgánicos al suelo genera efectos característicos sobre la disponibilidad de nutrientes y la actividad de la MA en el intercultivo maíz - soya. La práctica de $\mathrm{AO}$ hace más lento el proceso de mineralización de los compuestos orgánicos y genera, al mismo tiempo, imbibición de los nutrientes adicionados vía orgánica o sintética, lo cual favorece su liberación gradual. Estas cualidades permiten incrementos significativos en la actividad simbiótica del intercultivo con HMA.

La práctica de $\mathrm{AV}$ incrementa la velocidad de mineralización de los nutrientes en el suelo debido a que mejora el contacto de los residuos orgánicos con los microorganismos nativos, con sus consecuencias positivas sobre la disponibilidad de esos nutrientes. Sin embargo, esta disponibilidad no necesariamente va acompañada de la absorción radical eficiente de nutrientes, como el P vía HMA asociados al intercultivo.
La práctica de BA aporta poca biomasa y nutrientes al suelo, limitando su disponibilidad para el intercultivo. La adición de fertilizante de síntesis y compost al BA beneficia la nutrición del intercultivo pero reduce la participación de los HMA en la absorción de P. Esta disminución incide en mayor dependencia de insumos externos en el BA, reducción en la capacidad de retención de nutrientes y pérdida de estos por lixiviación y/o volatilización.

En maíz se alcanzaron mayores valores en las variables de HMA evaluadas (LME, \%MV y \% MEA) con respecto a la soya, y estos resultados pueden deberse al modelo fisiológico de las plantas (C4 en gramíneas), al igual que a la arquitectura del sistema radical que exhiben los dos cultivos, lo cual afecta la actividad de los HMA (Urcelay y Battistella, 2007; Parodi y Pezziani, 2011). Aunque la variabilidad espacial entre bloques jugó un papel importante en los resultados, también la falta de diferencias significativas de las variables de HMA entre tratamientos en ambos cultivos podría explicarse como incapacidad de estos hongos para distinguir entre calidad de materiales orgánicos y simplemente responder a su presencia, más aún cuando el suelo donde se hizo el ensayo se puede calificar como de mediana a alta fertilidad química. Los resultados podrían ser diferentes en otra condición edáfica. Sin embargo, la calidad de los residuos orgánicos utilizados se evidenció en forma significativa en los rendimientos en grano de los intercultivos, especialmente en soya. El análisis de componentes principales también mostró una relación estrecha entre rendimiento de grano con las variables de HMA evaluadas en soya y permitió observar la importancia de la interacción entre los componentes simbióticos MA y leguminosa - rizobios - MA cuando se busca la sostenibilidad de los agroecosistemas. 


\section{Conclusiones}

- Se observó mayor actividad de los HMA en maíz que en soya. El aporte de residuos vegetales de alta calidad procedentes del intercultivo maíz - frijol terciopelo, independiente de su forma de aplicación, y del barbecho procedente de Rottboelia cochinchinensis, estimula en forma similar la expresión de los componentes de HMA evaluados: LME, \%MV y \%MEA.

- La calidad de los residuos vegetales en la práctica agroecológica de acolchados orgánicos se reflejó en el rendimiento en grano seco del intercultivo, el cual fue similar al alcanzado bajo práctica convencional. Los efectos más notorios de esta práctica se observaron en el rendimiento en soya.

- El análisis de componentes permitió establecer que el AO individual y/o combinado con compost potencia el desarrollo del micelio externo vivo de HMA y su actividad sobre la absorción de $\mathrm{P}$ desde la solución del suelo, lo que finalmente se traduce en altos rendimientos de grano en maíz y soya en el suelo Typic Haplustert en el cual se hizo esta investigación.

\section{Agradecimientos}

Esta investigación fue posible gracias al apoyo de la Universidad Nacional de Colombia y del Departamento Administrativo de Ciencia y Tecnología de Colombia - COLCIENCIAS.

\section{Literatura citada}

Altieri, M.A.; Nicholls, C.I.

2012. Agroecología: Única esperanza para la soberanía alimentaria y la resiliencia sociecológica. Una contribución a las discusiones de Rio +20 sobre temas en la interface del hambre, la agricultura, y la justicia ambiental y social. Medellín, Colombia. 21 p.

Altieri, M.A.; Funes - Monzote, F.; Petersen, P.

2012. Agroecologically efficient agricultural systems for smallholder farmers: contributions to food sovereignty. Journal Agronomy for Sustainable Development, 32(1): 1-13.

Deguchi, S.; Shimazaki, Y.; Uozumi, S.; Tawaraya, K.; Kawamoto, H.; Tanaka, O.

2007. White clover living mulch increases the yield of silage corn via arbuscular mycorrhizal fungus colonization. Journal Plant Soil, 291: 291-299.

FENALCE. Federación Nacional de Cultivadores de Cereales y Leguminosas.

2013. Área, producción y rendimiento de cereales y leguminosas, semestre 2013A (Reporte 30 de junio). Bogotá, Colombia. 2 p.

Gómez Zambrano, J.

2011. Agricultura Hidrológica (Respuesta al cambio climático). Artículo publicado en las memorias del IV Encuentro Nacional de Agricultura de Conservación "Estrategias Tecnológicas para una Agricultura Ecoeficiente". Noviembre de 2011. Villavicencio Meta, Colombia. 10 p.

Gosling, P.; Hodge, A.; Goodlass, G.; Bending, G.D.

2006. Arbuscular mycorrhizal fungi and organic farming Agric. Journal Ecosystems Environment, 113: 17-35.

Ipsilantis, I.; Samourelis, C.; Karpouzas, D.

2012. The impact of biological pesticides on arbuscular mycorrhizal fungi. Journal Soil Biology and Biochemistry, 45: 147-155.
Juge, C.; Prevost, D.; Bertrand, A.; Bipfubusa, M.; Chalifour, F. P. 2012. Growth and biochemical responses of soybean to double and triple microbial associations with Bradyrhizobium, Azospirillum and arbuscular mycorrhizae. Journal Applied Soil Ecology, 61: 147-157.

Lau, J.A.; Bowling, E. J.; Gentry, L.E.; Glasser, P.A.; Monarch, E.A.; Olesen, W. E.; Waxmonsky, J.; Young, R.T.

2012. Direct and interactive effects of light and nutrients on the legume-rhizobia mutualism. Journal Acta Oecologica, 39: $80-86$

León, T.

2009. Agroecología: desafíos de una ciencia ambiental en construcción. Agroecología, 4 (1): 7-17.

Meghvansi, M. K.; Prasad, K.; Harwani, D.; Mahn, A.

2008. Response of soybean cultivars toward inoculation with three arbuscular mycorrhizal fungi and Bradyrhizobium japonicum in the alluvial soil. European Journal of soil biology, 44: 316-323.

Oehl, F.; Alves Da Silva, G.; Tomio, B.; Sieverding, E.

2011. Glomeromycota: three new genera and glomoid species reorganized. Journal Mycotaxon, 116: 75-120.

Ojiem, J.; Vanlauwe, B.; Ridder, N.; Giller, K.

2007. Niche-based assessment of contributions of legumes to the Nitrogen economy of Western Kenya smallholder farms. Journal Plant Soil, 292: 119-135.

Parodi, G.; Pezzani, F.

2011. Micorrizas arbusculares en dos gramíneas nativas de Uruguay en áreas con y sin pastoreo. Agrociencia, 15 (2): 1-10.

Prager, M.; Sanclemente, O. E.; Sánchez de Prager, M.; Gallego, J.M.; Ángel, D.I.

2012. Abonos verdes: Tecnología para el manejo agroecológico de los cultivos. Agroecología, 7: 53-62. 
Rosset, P.; Ávila, D.R.

2008. Causas de la crisis global de los precios de los alimentos, y la respuesta campesina. Ecología Política, 36: 18-22.

Salvagiotti. F.; Cassman. K.G.; Specht, J.E.; Walters, D.T; Weiss. A.; Dobermann. A.

2008. Nitrogen uptake, fixation and response to fertilizer $\mathrm{N}$ in soybeans: A review. Journal Field Crops Research, 108: 1-13.

Sánchez de Prager, M.; Gómez, E.D.; Muñoz, J.E.; Barrios, E.; Prager, M.; Bravo, N.; Mabrouk, E.S.; Pérez, J.; Azakawa, N.; Marmolejo, F.; Cadavid, L.F.; Quintero, R.; Miranda, J.C.; Mier, C.E.; Torres, R.; Trinidad, J.;Zapata, C.F.; Tofiño, R.; Benjumea, C.; Díaz, G.; Trujillo, L.; Bonilla, F.; Espinosa, J.C.; Rodríguez, H.; García, H.; Triana, W.; Carlosama, C.A.; Vargas, N.

2007. Las endomicorrizas: Expresión bioedáfica de importancia en el trópico. Universidad Nacional de Colombia Sede Palmira. Facultad de Ciencias Agropecuarias. Palmira, Colombia. $351 \mathrm{p}$.

Sánchez de Prager, M.; Narváez, M.; Vélez, F.; Ángel, D.I.; Vargas, N.

2009. La micorriza arbuscular (MA) como componente de estabilidad en los agroecosistemas. Rev. Bras. Agroecología, 4(2): 4.324-4.327.

Sánchez de Prager, M.; Posada, R.; Velásquez, D.; Narváez, M. 2010. Metodologías básicas para el trabajo con micorriza arbuscular y hongos formadores de micorriza arbuscular. Universidad Nacional de Colombia, Palmira. 139 p.
Sanclemente, O.E.; Prager, M.

2009. Efecto del cultivo de cobertura y abono verde: Мисиna pruriens en las propiedades biológicas de un suelo Typic Haplustalfs, cultivado con maíz dulce (Zea mays L.) en la zona de ladera del Municipio de Palmira Valle del Cauca, Colombia. Rev. Bras. Agroecología, 4(2): 4.133-4.138.

Sanclemente, O.E.

2015. Crisis de la agricultura convencional y agroecología como alternativa que aporta a la construcción de ciudadanía ambiental. En M. Cabrera (Ed.), Ciudadanía ambiental, crisis de la agricultura convencional y desafíos para una agroecología orientada hacia el desarrollo rural. Universidad Nacional Abierta y a Distancia. Bogotá, Colombia. Pp. 83-86.

Sieverding, E.

1991. Vesicular arbuscular Mycorrhiza management in tropical agroecosistem. GTZ Federal Republic of Germany, Berlim. $370 \mathrm{p}$.

Siqueira, J.O.

2010. Micorrizas: 30 años de pesquisas no Brasil. Editorial Universidade Federal de Lavras UFLA. Brasil. 716 p.

Tennant, D.

1975. A test of a modified line intersect method of estimating root length. Journal of Ecology, 63(3): 995-1.001.

Urcelay, C.; Battistella, R.

2007. Colonización micorrícica en distintos tipos funcionales de plantas herbáceas del centro de Argentina. Ecología Austral, 17: 179-188. 